\title{
The enthesis: a review of the tendon-to-bone insertion
}

\author{
John Apostolakos ${ }^{1}$ \\ Thomas JS Durant ${ }^{1}$ \\ Corey R. Dwyer ${ }^{1}$ \\ Ryan P. Russell ${ }^{1}$ \\ Jeffrey $H$. Weinreb ${ }^{1}$ \\ Farhang Alaee ${ }^{1}$ \\ Knut Beitzel ${ }^{2}$ \\ Mary Beth McCarthy ${ }^{1}$ \\ Mark P. Cote ${ }^{1}$
}

Augustus D. Mazzocca ${ }^{1}$

1 Department of Orthopaedic Surgery, University of Connecticut Health Center, Farmington, CT, USA

2 Department of Orthopaedic Sportsmedicine, Technical University Munich, Germany

\section{Corresponding author:}

Augustus D. Mazzocca

Department of Orthopaedic Surgery

University of Connecticut Health Center

Farmington, CT 06032, USA

Tel: 860-679-6709

Fax: 860-679-6649

E-mail: mazzocca@uchc.edu

\section{Summary}

The integration of tendon into bone occurs at a specialized interface known as the enthesis. The fibrous tendon to bone enthesis is established through a structurally continuous gradient from uncalcified tendon to calcified bone. The enthesis exhibits gradients in tissue organization classified into four distinct zones with varying cellular compositions, mechanical properties, and functions in order to facilitate joint movement. Damage to tendinous insertions is common in the field of orthopaedic medicine and often involves surgical intervention that requires the attempted recreation of the natural organization of tendon into bone. The difficulty associated with recreating the distinct organization may account for the surgical challenges associated with reconstruction of damaged insertion sites. These procedures are often associated with high failure rates and consequently require revision procedures. Management of tendinous injuries and recon- struction of the insertion site is becoming a popular topic in the field of orthopaedic medicine.

KEY WORDS: tendon, bone, enthesis, reconstruction.

\section{Introduction}

The robust musculoskeletal system provides support and stability to the human body and allows for the organized movement of the muscular and skeletal systems. The musculoskeletal system consists of bone, muscle, cartilage, tendon, ligament, and other connective tissues that organize the various components into a functional organ system. Connective tissues, such as tendons and ligaments, are joined to bone in a specialized interface referred to as an insertion site, known as the enthesis which integrates their structures into bone and facilitates joint motion ${ }^{1}$. The enthesis, which will be further discussed in subsequent sections of this review, exhibits gradients in tissue organization that are classified into four distinct zones of tissue with varying mechanical properties and functions $^{1,2}$. The distinct organization of this tendon/ligament to bone insertion site is essential to proper function of the respective muscular/skeletal structures and may account for the surgical challenges associated with reconstruction of damaged insertion sites.

Damage to connective tissues, specifically to tendons and ligaments, is common in the field of orthopaedic medicine and often requires operative intervention. The destruction of these tendinous and ligamentous structures impairs the natural specialized interface referred to as the enthesis and requires the surgeon to attempt to recreate the natural organization of the connective tissue insertion into the bone. Injuries such as rotator cuff and anterior cruciate ligament $(A C L)$ tears are relatively common and outcomes of these procedures serve as evidence of the difficulty in managing these injuries. These procedures are often associated with high failure rates and consequently require revision procedures. Studies report $11-95 \%$ failure rates in rotator cuff repair surgeries and that nearly half of all patients undergoing ACL reconstruction report unresolved pain one year postoperatively ${ }^{1,3-6}$.

Management of tendon/ligament to bone healing, and the reestablishment of the insertion site, is becoming an increasingly popular topic in the field of orthopaedic medicine. At the present time there is no 
optimal method for management of these injuries, however, several strategies are being actively discussed and researched. Current options, including mesenchymal stem cells (MSCs), bone marrow aspirate (BMA), growth factors, cell therapy, and platelet rich plasma (PRP), have shown promising results, but the development of a gold-standard for treatment remains in its infancy.

The purpose of this paper is 2-fold: (1) to explore the distinct tissue organization of the tendon-to-bone enthesis and its healing and (2) to describe the current strategies and associated outcomes related to the reconstruction of the tendon-to-bone enthesis, specifically regarding rotator cuff repairs. The authors acknowledge that information presented in this review has been obtained ethically and according to international standards as required by the Muscles, Ligaments, and Tendons Journal?.

\section{The insertion site or "enthesis"}

The enthesis is defined as the area where tendon, ligament, or joint capsule inserts into bone and acts to transmit tensile load from soft tissues to bone ${ }^{2}$. Entheses are critical as they allow for the proper transmission of contractile forces from the muscle belly to the respective skeletal attachment, while simultaneously dissipating force away from the enthesis itself, from tendon into bone ${ }^{1,8,9}$.

Entheses can be further described according to the type of tissue present at the skeletal attachment site, specifically, either dense fibrous connective tissue or fibrocartilage. Fibrous entheses attach directly to bone or periosteum primarily via fibrous tissue, which is similar in structure to the tendon midsubstance. Fibrocartilaginous entheses attach to bone through a layer of fibrocartilage which acts as a transition from the fibrous tendon tissue ${ }^{1,2,8}$. The majority of entheses in the body are fibrocartilaginous. These insertions are more commonly studied and more frequently injured (e.g. rotator cuff injuries) than fibrous insertions. Fibrocartilaginous insertions will therefore be the focus of this review. However, the authors will provide a brief overview of both types of entheses and the differences between them in order to provide the reader with a clinically relevant understanding of the two major forms of soft-tissue to bone insertions.

\section{Overview of fibrous and fibrocartilaginous entheses}

Fibrous and fibrocartilaginous entheses have been referred to in the literature as 'perioesteal-diaphyseal' and 'chondroapophyseal', or 'indirect' and 'direct', respectively². It is important to note that the terms 'indirect' for fibrous entheses and 'direct' for fibrocartilaginous entheses can be rather confusing. The 'indirect' description of the fibrous entheses, such as those found at the deltoid and pronator teres insertions, accurately emphasizes that at certain insertions, the tendon cannot directly insert to the bone but rather indirectly attach via the periosteum to ensure that the relative position is maintained during appositional growth ${ }^{2}$. These 'indirect' insertions are typically found at metaphyseal insertions. Using this description can be misleading as fibrocartilaginous entheses could also be considered 'indirect' as their attachment to bone is indirect via zones of fibrocartilage and therefore not 'direct' attachments by the fibrous tissue ${ }^{2}$. This review will refer to the two major entheses as fibrous and fibrocartilaginous (Tab. 1).

\section{Fibrous entheses}

Fibrous entheses are characterized by dense fibrous connective tissue at the tendon-bone interface and are common in tendons that attach to diaphyses of long bones $^{8}$. These entheses typically occur over large surface areas and are characterized by perforating mineralized collagen fibers ${ }^{1}$. Furthermore, these entheses can be either 'bony' or 'periosteal' depending on whether the tendon inserts directly into bone or periosteum respectively ${ }^{8}$. This type of enthesis is found in muscles such as the deltoid, which inserts into the humerus, and the muscles attaching to the linea aspera of the femur, such as the adductor magnus ${ }^{1,9}$. Fibrous entheses have received relatively little attention in the literature compared to fibrocartilaginous entheses, likely due to the fact that overuse injuries are more common in fibrocartilaginous tendon-to-bone insertions such as those of the rotator cuff (Figs. 1, 2).

\section{Fibrocartilaginous entheses}

Fibrocartilaginous entheses are characterized by fibrocartilage at the tendon-bone interface and are typical of epiphyses and apophyses ${ }^{8}$. These types of en-

Table 1. Characteristics of fibrous and fibrocartilaginous entheses.

\begin{tabular}{lll}
\hline & Fibrous Entheses & Fibrocartilaginous Entheses \\
\hline Common Attachment & Metaphyses and Diaphyses of long bones (7) & Epiphyses and Apophyses $(7)$ \\
\hline Composition & Perforating mineralized collagen fibers (1) & Four distinct zones $(1,7)$ \\
\hline Angle of Insertion & $\begin{array}{l}\text { Insertion angle changes slightly during } \\
\text { motions (2) }\end{array}$ & $\begin{array}{l}\text { Prone to overuse injuries as the insertion } \\
\text { angle changes are greater }(2)\end{array}$ \\
\hline Example & $\begin{array}{l}\text { Deltoid attachment to the humerus and } \\
\text { Adductor magnus to the linea aspera } \\
\text { of the femur, pronator teres }(1,8)\end{array}$ & Rotator Cuff and Achilles Tendons $(1,7)$ \\
\hline
\end{tabular}



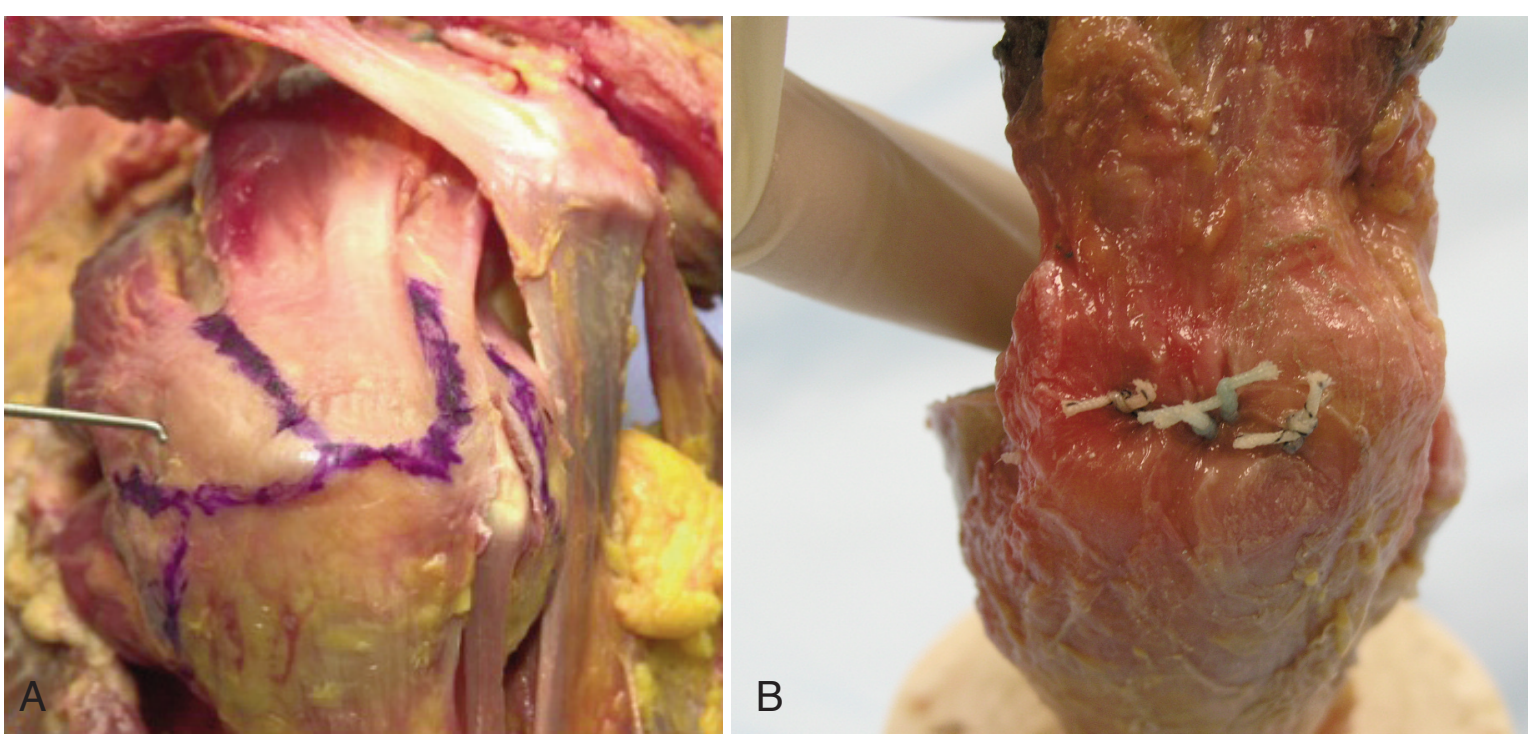

Figure 1. Cadaveric image of A) an intact rotator cuff, B) a repaired rotator cuff.

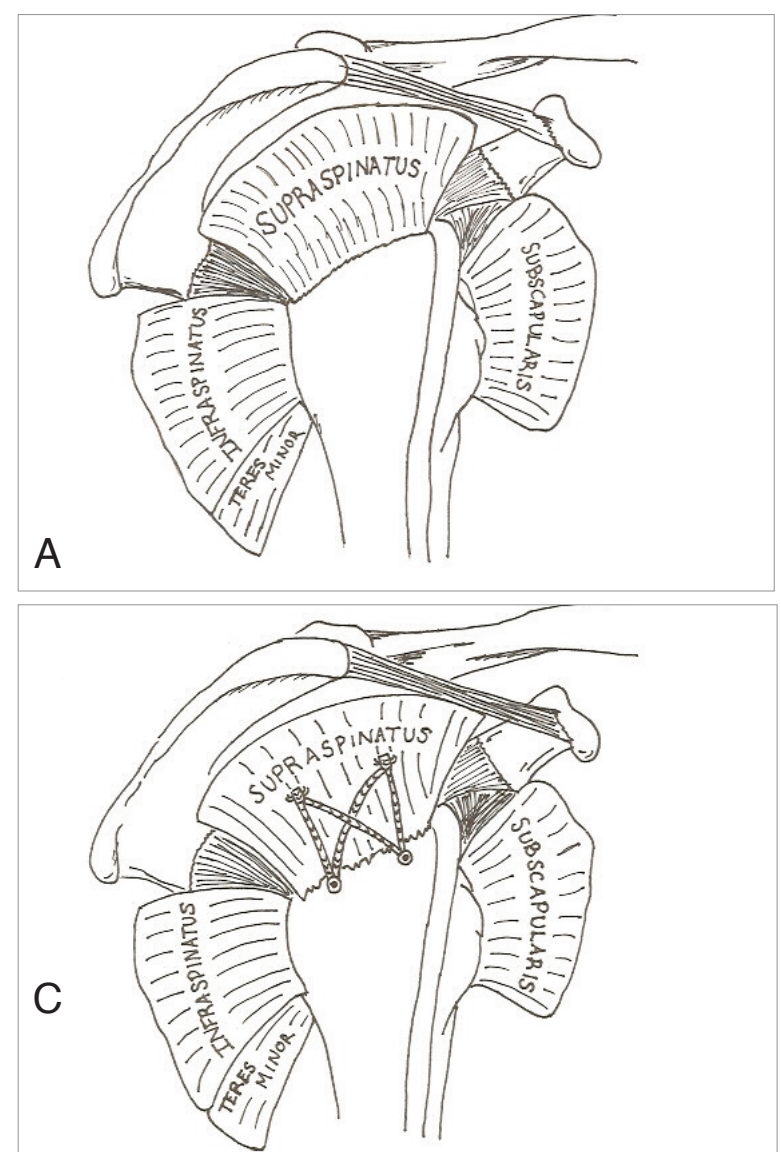

theses are more common than fibrous entheses and are prone to overuse injuries such as those of the rotator cuff and Achilles tendons ${ }^{1,8}$. A typical fibrocartilaginous enthesis has four distinct zones that create a structurally continuous gradient from uncalcified tendon to calcified bone ${ }^{1,8}$. These zones, in order,

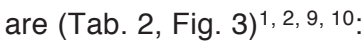

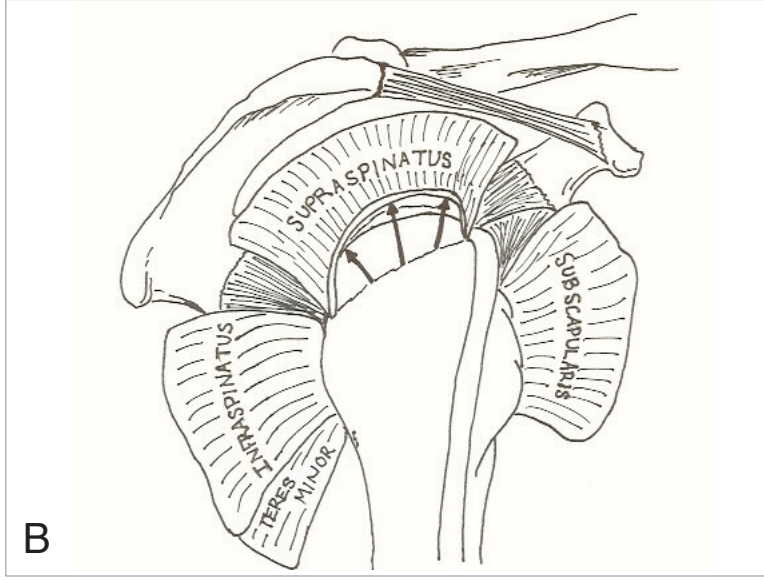

Figure 2. Illustrations of $A$ ) an intact supraspinatus tendon of the rotator cuff, B) a retracted tear in the supraspinatus tendon of the rotator cuff, C) a repaired tear in the supraspinatus tendon of the rotator cuff.

\section{1) Pure dense fibrous connective tissue}

Pure dense fibrous connective tissue is composed of pure tendon and is heavily populated by fibroblasts ${ }^{1}$. The mechanical properties of this zone are similar to those of mid-substance tendon, with its composition consisting mainly of linearly arranged type I collagen 


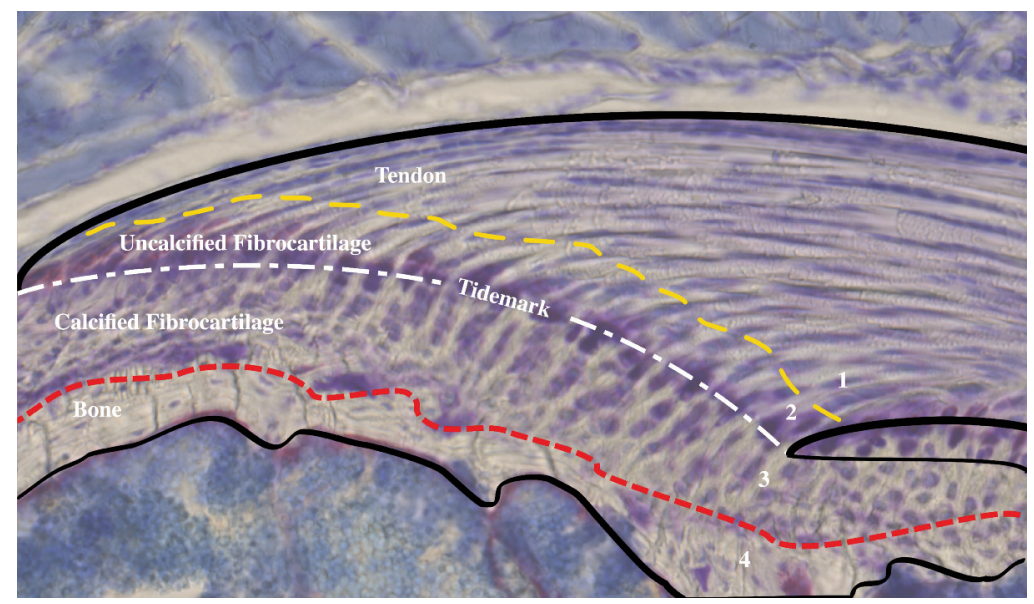

Figure 3. Illustration of the four zones of the enthesis superimposed on a histological section of a mouse supraspinatus. Proteoglycans in tendon, fibrocartilage and calcified fibrocartilage are seen in purple after staining with toluidine blue, which highlights the compositional gradient characteristic of the enthesis.

as well as some type III collagen, elastin, and proteoglycans within the ground substance surrounding the cells ${ }^{1,9,11,12}$.

\section{2) Uncalcified fibrocartilage}

Uncalcified fibrocartilage is an avascular zone of uncalcified, or unmineralized, fibrocartilage populated by fibrochondrocytes and consisting of the proteoglycan aggrecan and types I, II, and III collagen 1, 2, 7, 9, 11-14. In addition to the specific cellular composition of the uncalcified fibrocartilage zone, it is also important to note the mechanical functions the zone has on fibrocartilaginous insertions such as that of the rotator cuff. In most long bones, fibrocartilaginous insertions are found on the epiphyses and apophyses in contrast to the fibrous insertions routinely found on meta- physes and diaphyses ${ }^{2,15}$. The differences in the relative positions of these insertions has an important impact on the mechanical function of the uncalcified fibrocartilage zone of the fibrocartilaginous entheses when considering the insertion angle of the tendonbone insertion ${ }^{2}$. To better illustrate this functional difference, consider the fibrous insertion of the deltoid tendon into the humerus in comparison to the fibrocartilaginous insertion of the supraspinatus tendon of the rotator cuff. During abduction of the arm, the insertion angle of the tendon into the bone changes only slightly for the deltoid tendon as compared to the angle of the supraspinatus tendon, therefore making the latter insertion more prone to injury from chronic use $^{2}$. Fibrocartilaginous entheses are more disposed to wear and tear at the insertion site due to their location and function in the body. The uncalcified fibrocar-

Table 2. Zones of fibrocartilaginous entheses.

\begin{tabular}{|c|c|c|}
\hline & Composition & Significance \\
\hline $\begin{array}{l}\text { Zone } 1 \\
\text { Pure Dense Fibrous } \\
\text { Connective Tissue }\end{array}$ & $\begin{array}{l}\text { Fibroblasts } \\
\text { Type I Collagen } \\
\text { Type III Collagen }(1,8,10,11)\end{array}$ & $\begin{array}{l}\text { Linearly arranged collagen with mechanical } \\
\text { properties similar to mid-substance tendon } \\
(1,8,10,11)\end{array}$ \\
\hline $\begin{array}{l}\text { Zone } 2 \\
\text { Uncalcified Fibrocartilage }\end{array}$ & $\begin{array}{l}\text { Fibrochondrocytes } \\
\text { Proteoglycan aggrecan with } \\
\text { Collagen (Types I-III) }(1,2,8,10-14)\end{array}$ & Dissipates bending of collagen fibers in tendon (2) \\
\hline Tidemark & & $\begin{array}{l}\text { Basophilic demarcation between uncalcified and } \\
\text { calcified fibrocartilage representing the boundary } \\
\text { between soft and hard tissues (8) }\end{array}$ \\
\hline $\begin{array}{l}\text { Zone } 3 \\
\text { Calcified Fibrocartilage }\end{array}$ & $\begin{array}{l}\text { Fibrochondrocytes } \\
\text { Type II Collagen (Predominant) } \\
\text { Type I Collagen } \\
\text { Type X Collagen }(1,2,3,10-14)\end{array}$ & $\begin{array}{l}\text { Irregularity of attachments into bone give } \\
\text { mechanical integrity of enthesis (2) }\end{array}$ \\
\hline $\begin{array}{l}\text { Zone } 4 \\
\text { Bone }\end{array}$ & $\begin{array}{l}\text { Osteocytes } \\
\text { Osteoblasts } \\
\text { Osteoclasts }(1,8) \\
\text { Type I Collagen }\end{array}$ & Provides sites of attachment for the tendon \\
\hline
\end{tabular}


tilage zone functions as a force damper to dissipate stress generated by bending collagen fibers in the tendon ${ }^{2}$. The functional impact of this zone is supported by studies reporting that the quantity of uncalcified fibrocartilage is increased at insertion sites with more variable ranges of insertion angles during joint movements ${ }^{2,16}$.

\section{Tidemark}

The tidemark is a basophilic line that separates the uncalcified and calcified fibrocartilage zones. This is more clearly described as the mechanical boundary between soft and hard tissues ${ }^{9}$. The tidemark is relatively straight which indicates the production of a flat surface during the mineralization process which is important clinically as this surface reduces the risk of damage to soft tissues during joint movement ${ }^{9}$.

\section{3) Calcified fibrocartilage}

Calcified fibrocartilage is an avascular zone of calcified, or mineralized, fibrocartilage populated by fibrochondrocytes and consisting of predominantly type II collagen as well as aggrecan and types I and X collagen ${ }^{1,2}$, $7,9,11-14$. This zone represents the true junction of the tendon to the bone and creates a boundary with the subchondral bone ${ }^{2}$. In contrast to the tidemark, this anatomical junction of tendon to bone is highly irregu$\operatorname{lar}^{2}$. This irregularity is functionally important as the attachments of the calcified fibrocartilage layer into the bone provide the mechanical integrity of the enthesis ${ }^{2}$. Several studies investigating the 3D modeling of fibrocartilaginous entheses report there is a considerable amount of interlocking between the calcified fibrocartilage zone and bone which further suggests the functional importance of this zone ${ }^{2}$.

Certain investigators stress the importance of distinct anatomical and mechanical boundaries of the insertion with a thin layer of calcified fibrocartilage separating the two. This layer is believed to be important in allowing a gradual transition of force across the enthesis in addition to acting as a barrier against blood vessels in the bone and preventing direct cell-cell communication between osteocytes and tendon cells $^{8}, 17,18$. Studies performed by Benjamin et al. ${ }^{8,18-}$ 20 suggest that this boundary may reduce the risk of infection spreading from the more highly vascularized bone into tendon. These studies acknowledge that the full significance of these barriers between cell-cell communication and blood vessels is not completely understood but note it may be related to preventing bone tissue development into adjacent tendon as bony spurs typically develop in the most fibrous regions of fibrocartilaginous entheses.

\section{4) Bone}

Bone consists of osteoclasts, osteocytes, and osteoblasts residing in a matrix of type I collagen and carbonated apatite mineral ${ }^{1,}, 9$.

\section{Tendon insertion healing}

In contrast to the organized, distinct development of the four zones of the cartilaginous enthesis, tendonbone healing occurs through the formation of $\mathrm{fi}$ brovascular scar tissue and does not reestablish the native tendon-bone insertion site formed during embryological development ${ }^{1}, 9,14,21-23$. The work done by Thomopoulos et al. ${ }^{24}$ stresses the importance of understanding the development and morphogeneses of the tendon-bone enthesis in order to more fully understand the healing process of the insertion site. Interestingly, the work of several investigators acknowledges that both biological and mechanical factors are vital to the proper development of the enthesis $25-27$. The basis of the work of these investigators is that an understanding of the natural development of the tendon to bone insertion site allows investigators to research and design biological and mechanical strategies to reestablish the native enthesis and determine potential treatments for tendon-bone-healing problems currently faced in the field of orthopaedic medicine.

Thomopoulos et al. ${ }^{24}$ report it's likely that the gradations in biological factors of the tendon-bone enthesis promote gradations in cell differential and subsequent tissue formation. Additionally, mechanical load during development of entheses is vital to mineral accumulation, fibrocartilage formation, and collagen fiber formation necessary for proper generation of the insertion site ${ }^{24}$. The need for both biological factors and mechanical influences on the development of entheses in combination with the specific composition, function, and mechanical properties of the four zones of fibrocartilaginous entheses makes it extremely difficult to recreate this native insertion.

Several studies have reported that the gradations developed in native tendon are not regenerated during tendon to bone healing 14, 21-23, 28-32. Furthermore, the resulting fibrovascular scar tissue interface has been shown to be mechanically weaker and more prone to failure than that of the natural tendon-bone enthesis created during prenatal development ${ }^{9}, 24,28,33$. At the present time, little is known about the natural healing process of the tendon-bone enthesis making it difficult to develop an effective treatment option. In a study on a rat rotator cuff model, Thomopoulos et al. ${ }^{14}$ reported healing sites with excessive scar formation and a lack of fibrocartilage formation at the tendon-bone enthesis ${ }^{28}$.

From what is known at the current time, development of this fibrovascular scar tissue has been reported to occur in three stages: inflammation (0-7 days), repair (5-14 days), and remodeling (>14 days) ${ }^{9}, 10,33$. The inflammatory stage begins with platelets depositing fibrin and fibronectin leading to the accumulation of macrophages in response to insulin-like growth factor 1 (IGF-1), platelet-derived growth factor (PDGF), and transforming growth factor $\beta(\text { TGF }-\beta)^{9}$. TGF- $\beta$ has been associated with scar and adhesion formation in several studies and may account for the production of 
scar tissue in the regeneration of fibrocartilaginous entheses such as the rotator cuff28, 34-36. Of the three mammalian forms of TGF- $\beta$, TGF- $\beta 1$ and TGF- $\beta 3$ are believed to be important for musculoskeletal growth and differentiation, making them critical factors in the healing process of the tendon-bone enthesis ${ }^{28,36,37 .}$ TGF- $\beta 1$ is typically found during all stages of adult wound healing of these tissues and is associated with cell migration and angiogenesis whereas TGF- $\beta 3$ is found in adult articular cartilage and during scarless fetal wound healing $27,28,36,38-41$. The transition to the repair phase occurs when macrophages begin to secrete TGF- $\beta 1$, which results in fibroblastic proliferation and formation of scar tissue ${ }^{9}$. This scar tissue is primarily composed of type III collagen and undergoes matrix metalloproteinase (MMP) mediated remodeling as result of the turnover of the extracellular matrix $^{9,40}$.

An additional aspect of tendon degeneration and healing involves the differences in acute inflammatory versus chronic overuse injury models. The work of Cook and Purdam ${ }^{42}$ describes a continuum of tendon pathology that explains the clinical presentation of load-induced tendinopathy. The proposed continuum occurs in the following 3 stages:

1) Reactive Tendinopathy - This stage involves a non-inflammatory proliferative response in the cell and matrix which may occur after an acute tensile or compressive load. Clinically, this stage typically results from an acute overload after physical activity and leads to a short term response that thickens the tendon, reduces stress, and increases stiffness.

2) Tendon Dysrepair - This is the stage of attempted tendon healing characterized by an increase in chrondrocytic and myofibroblast cells resulting in increased protein production (proteoglycan and collagen). This increase results in separation of the collagen and disorganization of the matrix.

3) Degenerative Tendinopathy - This stage includes both matrix and cellular changes with increased acellularity. The increased areas of cell death lead to an unorganized matrix with decreased collagen content that has little capacity for reversible changes.

Despite the proposed continuum and its relation to overuse injury, pathogenic mechanisms of tendinopathies remain largely unknown ${ }^{43}$. Specifically, the contribution of inflammation and degeneration to chronic pain in tendinitis remains in question. Tendinitis is commonly used to describe pain referring to a symptomatic tendon with the underlying pathological process involving inflammation ${ }^{43}$. In contrast to the theory of inflammatory causes of chronic tendon pain is the limited success of treatments aimed at diminishing inflammation while other histological studies have reported the presence of degenerative lesions with minimal inflammation ${ }^{17,43-45}$. Based on the difficulty associated with determining the presence and contributions of inflammation and degeneration in a tendon injury, the terms 'tendinosis' or 'tendinopathy' have gained increasing popularity ${ }^{3}, 43$.

\section{Current strategies for augmentation}

Largely due to the functional importance of the tendon-bone enthesis, research aimed at the regeneration of the embryonically developed interface is a rapidly evolving field of orthopaedic medicine. Major challenges to improving the ability to reestablish this tendon-bone interface are the identification of the optimal healing factors, timing of delivery of these healing factors, and application of these factors to the site of repair through the use of scaffolds. More specifically, attempts to recreate the tendon-bone enthesis are extremely difficult because of the need to pre-engineer the complex tissue gradient of the native tendon ex vivo followed by the need to apply these healing factors to the insertion site in vivo. Therefore, regeneration of the native tendon-bone interface requires the complex and not completely understood combination of cell-and scaffold-based approaches to successfully recreate this complex interface. Despite the lack of a complete understanding of the biological factors associated with natural tendon to bone healing, several investigators are pursuing promising strategies in an attempt to determine a treatment to most closely reestablish the tendon to bone enthesis. Augmentation of the bone-tendon interface using MSCs is an experimental strategy being used in attempt to regenerate the native enthesis. The term "stem cells" refers to unspecialized cells that have the ability to provide a self-renewing population and have the ability to differentiate into various adult stem cells ${ }^{5}$. Multipotent adult stem cells are described as having the differentiation potential limited to one germ layer. If the stem cells have the potential to differentiate into mesencyhmal tissue (e.g. bone, tendon, cartilage, muscle) they are classified as $\mathrm{MSCs}^{5}$. The selfrenewing and multipotent potential of MSCs in combination with their ability to produce growth factors and cytokines make the use of MSCs a promising strategy for the regeneration of the tendon-bone enthesis ${ }^{5,} 41$, 46-48. A recent review performed by Beitzel at al. ${ }^{5}$ reviewed the use of MSCs to augment shoulder disorders and concluded that the limited state of the literature reports promising approaches to enhancing tendon-bone healing methods, however the use of MSCs for shoulder surgery at the present time should be considered an experimental technique demonstrating the need for further research.

In addition to the cell therapy strategies being researched, several investigators are looking into the efficacy of growth factors in enthesis regeneration. As described previously, the inflammatory stage of the tendon healing process occurs as the result of signaling directed by cytokines, or growth factors, including PDGF and TGF- $\beta^{9,49}$. These growth factors are essential for cell chemotaxis, proliferation, differentiation, and extracellular matrix synthesis during the healing process ${ }^{9}$. Based on the critical roles of these growth factors, addition of these cytokines offers the potential to successfully augment the tendon to bone enthesis repair. PDGF is a basic protein family and 
exists in three isoforms (PDGF-AA, PDGF-BB, PDGF-AB) which function as chemotactic agents for inflammatory cells and assist in increasing composition of type I collagen synthesis while inducing TGF$\beta 1^{9}, 50$. PDGF-BB has been the subject of the most research as this isoform stimulates both extracellular matrix synthesis and cell division ${ }^{9}, 51,52$. Applying PDGF-BB to a polyglycolic acid (PGA) scaffold, Uggen et al. ${ }^{52}$ reported regeneration of normal crimp patterning and collagen-bundle alignment in a rat rotator cuff model. In addition to the use of PDGA, TGF- $\beta$ is also considered an important cytokine in the restoration of the tendon to bone enthesis. Early studies reporting on the application of TGF- $\beta 3$ in rat rotator cuff models have shown promising results including accelerated healing, increased cell proliferation, vascularity, bone formation, fibrocartilage, and improved collagen organization ${ }^{1,}$, 9, 53-55.

In contrast to strategies based on healing models via inflammation, other investigators report on the changes in growth factor signaling in cases of chronic tendon degeneration with little inflammation. Fenwick et al. ${ }^{4}$ reported on the expression of TGF- $\beta$ isoforms in patients with chronic tendinosis of the Achilles tendon. Based on the strong association between TGF- $\beta$ and tissue repair, the study investigated the expression of TGF isoforms $\beta 1, \beta 2$, and $\beta 3$ and their signaling receptors TGF- $\beta$ R1 and TGF- $\beta$ RII in both normal and pathological tendons ${ }^{4}$. The authors reported that cells in the matrix of normal tendon showed staining for TGF- $\beta 2$ yet displayed no staining for TGF- $\beta 1$ or TGF- $\beta 3$. In comparison, the pathological tendon showed an increase in cell numbers, an increase in the percentage of TGF- $\beta 2$ expression, and an increase in the number of cells expressing TGF- $\beta$ RII ${ }^{4}$. The investigators noted an evident lack of type 1 TGF- $\beta$ receptors at the level of antibody detection in pathological tendon suggesting the absence or low level expression of these receptors. Prior investigators have reported that TGF- $\beta$ requires both type 1 and 2 receptors for propagation of its signal and therefore the lack of type 1 receptors in this investigation suggest that proper signaling may not be induced in cases of chronic degradation even in the presence of active TGF- $\beta^{4,11,12}$. Results of the investigation may suggest that the treatment of chronic tendinopathy with exogenous TGF- $\beta$ may not have an effect based on the absence of type I TGF- $\beta$ receptors ${ }^{4}$.

Further complicating the issues surrounding the exogenous application of cytokines and growth factors is the limited clinical generalizability of data due to the differing mechanisms of acute injury versus chronic overuse injury ${ }^{13}$. Scott et al. ${ }^{13}$ reported on the extent of proliferation and apoptosis in an overuse tendinosis model of a rat supraspinatus tendon. The authors concluded that early tendinosis was associated with local stimulation of tenocytes in the absence of inflammation or apoptosis. Results of the study indicated that the early-stage upregulation of IGF-1 plays an important role in the process of load-induced tenocyte responses during pathogenesis of overuse tendon injuries. Although cell death was not identified in the study, the investigators acknowledge the reported role of apoptosis in the process of overuse tendon injury and concluded that the findings of the study are not consistent with cell death in the initial stages of tendinosis ${ }^{13}$. Furthermore, the load-dependent cellular responses appear to predominate in early stages providing a possible explanation for the thickened appearance of many tendinosis lesions. Despite this initial stage of hypercellularity, apoptosis may play a role in more advanced stages of chronic overuse injury where applications of exogenous factors to promote cellular proliferation may be more appropriate. The differences in cellular responses of acute versus chronic overuse injuries are important to consider and may affect clinical decision making in terms of when to appropriately apply exogenous factors to the site of injury. The development of an effective augmentation strategy is made increasingly difficult because of the need to produce a sturdy and reliable exogenous carrier of these growth factors and cells to ensure direct placement of these sources to the repair site (Fig. 4). At the current time, questions still exist regarding optimal delivery carriers, timing of delivery, and dosing/combinations of exogenous factors. Theoretically, an ideal scaffold would effectively integrate with the host environment and consist of exogenously prepared gradients to restore the native tissue organization and mechanical properties of the normal insertion site $^{9}$. Specific descriptions of the various scaffolds is considered beyond the scope of this review, however a brief overview of the current state of the literature will be covered here.

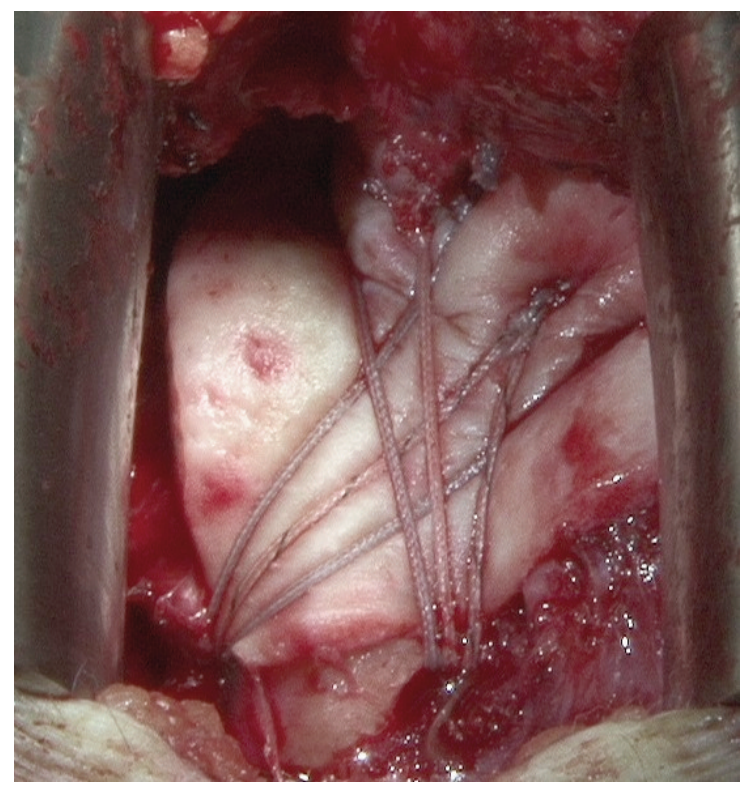

Figure 4. Intraoperative image of a revision open rotator cuff repair. Image portrays augmentation technique utilizing an acellular matrix patch soaked in autologous conditioned plasma (ACP). 
Scaffolds must be designed to support the distinct yet structurally continuous gradients of the fibrocartilaginous insertion in order to replicate the specific tissue organization and mimic the mechanical properties of the native enthesis. These scaffolds, described as stratified or multiphasic, are currently being studied in order act as carriers of specific cell therapies and growth hormones which should exhibit the phase specific composition and organization of the native enthesis and ultimately mimic the mechanical properties of the enthesis when incorporated into the repaired insertion site in vivo ${ }^{1}$. Based on the importance of the specific organization of the natural enthesis, an important aspect of the design is the production of these phase specific gradients into a structurally continuous scaffold. Another key design aspect is to incorporate distinct organization and interactions of specific cell populations which need to be monitored in order to most effectively reestablish normal formation, homeostasis, and repair of these insertion sites ${ }^{1}$. Finally, biological integration of the scaffold into the bone must be considered in order to complete fixation.

Ultimately, the use of cell therapy, growth factors, and scaffolds is a continuing topic of orthopaedic medicine and will need to be continued. Although the state of enthesis regeneration remains in its infancy, the future of this field is promising. Moffat et al. ${ }^{56}$ reported using a biphasic scaffold of polylactide-co-glycolide (PLGA) nanofibers and a composite of PLGA nanofibers and hydroxyapatite nanoparticles in the first and second phases respectively ${ }^{9,57}$. The study found that nanofiber organization has a significant effect on human rotator cuff fibroblast response and that physiologically relevant mechanical properties were maintained in vitro ${ }^{56}$. The findings showed that the biphasic scaffold was capable of promoting the formation of uncalcified and calcified matrix regions and acknowledged the need for future work focusing on scaffold optimization in vivo ${ }^{9,56}$.

Of more recent consideration is the role of gene therapy in tendon injury. A 2013 review by Juneja and Veillette ${ }^{14}$ summarizes the state of the literature regarding genetic alterations and knockdown approaches aimed at accessing the role of key proteoglycans and glycoproteins in the structural development, function, and repair of tendon, ligament, and enthesis. Although a thorough description of the current status of this research is outside the scope of this review, the basis of this research is to gain a more comprehensive understanding of the genes for proteoglycans and glycoproteins which affect not only the development and structure of tendons and ligaments but also the various aspects of their mechanical and viscoelastic properties as well as the phases of their healing processes ${ }^{14}$. An understanding of genes involved in these processes represent potential drug targets for pathological mechanisms that lead to various tendoninous abnormalities and may provide more options for therapeutic intervention in the future.

\section{Conclusion}

Tendon/ligament-to-bone entheses are critical components of the musculoskeletal system as they allow for the proper transmission of forces from muscle into bone. Injuries to the tendon/ligament-to-bone enthesis are common in the field of orthopaedic medicine yet high failure rates are often associated with their repair. In order to better treat these injuries it is important to understand the distinct organization and composition of these insertion sites. This review focuses on tendonbone entheses and, more specifically, the distinct yet structurally continuous fibrocartilaginous entheses such as the insertions of the rotator cuff tendons. Fibrocartilaginous entheses consist of four distinct zones ranging from uncalcified tendon to calcified bone, with each zone consisting of varying cell types, extracellular composition, and mechanical functions. In contrast to the distinct organization of natural tendon-bone insertions, tendon-bone healing is not completely understood at the present time but is characterized by the formation of fibrovascular scar tissue that is mechanically weaker and more prone to failure. Based on the structural importance of fibrocartilaginous entheses, strategies aimed at the regeneration of the tendonbone enthesis is a rapidly evolving field of orthopaedic medicine with current strategies including the use of cell therapy, growth factors, and scaffolds. At the present time, major challenges exist regarding the identification of optimal healing factors, timing and delivery of these healing factors, and application of these factors to the repair site. Additional challenges include the differences in cellular responses to acute versus chronic overuse tendon injuries which further complicates clinical determination of appropriate factors to administer at specific time periods. Despite the promising future of these therapies, regeneration of the tendon-bone enthesis remains in its infancy and requires future research aimed at discovering solutions to the challenges presented in this review.

\section{Disclosures}

Dr. Augustus D. Mazzocca is a paid consultant for Arthrex, Inc. Naples, FL, USA.

\section{Acknowledgements}

The authors thank Dr. Nathaniel Dyment for assistance with the histology for the enthesis zone illustration.

\section{References}

1. Lu HH, Thomopoulos S. Functional attachment of soft tissues to bone: development, healing, and tissue engineering. Annu Rev Biomed Eng. 2013;15:201-226.

2. Benjamin M, Ralphs JR. Entheses-the bony attachments of tendons and ligaments. Ital J Anat Embryol. 2001;106(2 Suppl 1):151-157. 
3. Maffulli N, Khan KM, Puddu G. Overuse tendon conditions: time to change a confusing terminology. Arthroscopy. 1998;14(8):840-843.

4. Fenwick SA, Curry V, Harrall RL, Hazleman BL, Hackney R, Riley GP. Expression of transforming growth factor-beta isoforms and their receptors in chronic tendinosis. J Anat. 2001;199(Pt 3):231-240.

5. Beitzel K, Solovyova O, Cote MP, et al. The future role of mesenchymal stem cells in the management of shoulder disorders. Arthroscopy. 2013;29(10):1702-1711.

6. Lafosse L, Brozska R, Toussaint B, Gobezie R. The outcome and structural integrity of arthroscopic rotator cuff repair with use of the double-row suture anchor technique. J Bone Joint Surg Am. 2007;89(7):1533-1541.

7. Padulo J, Oliva F, Frizziero A, Maffulli N. Muscles, Ligaments and Tendons Journal. Basic principles and recommendations in clinical and field science research. MLTJ. 2013;4:250-252.

8. Benjamin M, Kumai T, Milz S, Boszczyk BM, Boszczyk AA, Ralphs JR. The skeletal attachment of tendons-tendon "entheses". Comp Biochem Physiol A Mol Integr Physiol. 2002;133(4):931-945.

9. Angeline ME, Rodeo SA. Biologics in the management of rotator cuff surgery. Clin Sports Med. 2012;31(4):645-663.

10. Gulotta LV, Rodeo SA. Growth factors for rotator cuff repair. Clin Sports Med. 2009;28(1):13-23.

11. Laiho M, Weis MB, Massague J. Concomitant loss of transforming growth factor (TGF)-beta receptor types I and II in TGF-beta-resistant cell mutants implicates both receptor types in signal transduction. J Biol Chem. 1990;265(30):1851818524.

12. Wrana JL, Attisano L, Wieser R, Ventura F, Massague J. Mechanism of activation of the TGF-beta receptor. Nature. 1994;370(6488):341-347.

13. Scott A, Cook JL, Hart DA, Walker DC, Duronio V, Khan KM. Tenocyte responses to mechanical loading in vivo: a role for local insulin-like growth factor 1 signaling in early tendinosis in rats. Arthritis Rheum. 2007;56(3):871-881.

14. Juneja SC, Veillette $C$. Defects in tendon, ligament, and enthesis in response to genetic alterations in key proteoglycans and glycoproteins: a review. Arthritis. 2013;2013:154812.

15. Benjamin M, Evans EJ, Copp L. The histology of tendon attachments to bone in man. J Anat. 1986;149:89-100.

16. Benjamin M, Ralphs JR. Fibrocartilage in tendons and ligaments - an adaptation to compressive load. J Anat. 1998;193 ( Pt 4):481-494.

17. Alfredson $\mathrm{H}$, Ljung $\mathrm{BO}$, Thorsen $\mathrm{K}$, Lorentzon $\mathrm{R}$. In vivo investigation of ECRB tendons with microdialysis technique-no signs of inflammation but high amounts of glutamate in tennis elbow. Acta Orthop Scand. 2000;71(5):475-479.

18. Benjamin M, McGonagle D. Entheses: tendon and ligament attachment sites. Scand J Med Sci Sports. 2009;19(4):520527.

19. Benjamin M, Kaiser E, Milz S. Structure-function relationships in tendons: a review. J Anat. 2008;212(3):211-228.

20. Benjamin M, Toumi H, Suzuki D, Hayashi K, McGonagle D. Evidence for a distinctive pattern of bone formation in enthesophytes. Ann Rheum Dis. 2009;68(6):1003-1010.

21. Rodeo SA, Arnoczky SP, Torzilli PA, Hidaka C, Warren RF Tendon-healing in a bone tunnel. A biomechanical and histological study in the dog. J Bone Joint Surg Am. 1993;75(12):1795-1803.

22. Fujioka H, Thakur R, Wang GJ, Mizuno K, Balian G, Hurwitz SR. Comparison of surgically attached and non-attached repair of the rat Achilles tendon-bone interface. Cellular organization and type $\mathrm{X}$ collagen expression. Connect Tissue Res. 1998;37(3-4):205-218

23. Aoki M, Oguma H, Fukushima S, Ishii S, Ohtani S, Murakami G. Fibrous connection to bone after immediate repair of the ca- nine infraspinatus: the most effective bony surface for tendon attachment. J Shoulder Elbow Surg. 2001;10(2):123-128.

24. Thomopoulos S, Genin GM, Galatz LM. The development and morphogenesis of the tendon-to-bone insertion - what development can teach us about healing. J Musculoskelet Neuronal Interact. 2010;10(1):35-45.

25. Schweitzer R, Chyung JH, Murtaugh LC, et al. Analysis of the tendon cell fate using Scleraxis, a specific marker for tendons and ligaments. Development. 2001;128(19):3855-3866.

26. Thomopoulos S, Kim HM, Rothermich SY, Biederstadt C, Das $\mathrm{R}$, Galatz LM. Decreased muscle loading delays maturation of the tendon enthesis during postnatal development. J Orthop Res. 2007;25(9):1154-1163.

27. Kim HM, Galatz LM, Patel N, Das R, Thomopoulos S. Recovery potential after postnatal shoulder paralysis. An anima model of neonatal brachial plexus palsy. J Bone Joint Surg Am. 2009;91(4):879-891.

28. Galatz LM, Sandell LJ, Rothermich SY, et al. Characteristics of the rat supraspinatus tendon during tendon-to-bone healing after acute injury. J Orthop Res. 2006;24(3):541-550.

29. Fujioka H, Wang GJ, Mizuno K, Balian G, Hurwitz SR. Changes in the expression of type-X collagen in the fibrocartilage of rat Achilles tendon attachment during development. J Orthop Res. 1997;15(5):675-681.

30. Silva MJ, Boyer MI, Ditsios K, et al. The insertion site of the canine flexor digitorum profundus tendon heals slowly following injury and suture repair. J Orthop Res. 2002;20(3):447-453.

31. St Pierre P, Olson EJ, Elliott JJ, O'Hair KC, McKinney LA, Ryan $\mathrm{J}$. Tendon-healing to cortical bone compared with healing to a cancellous trough. A biomechanical and histological evaluation in goats. J Bone Joint Surg Am. 1995;77(12):1858-1866.

32. Thomopoulos S, Hattersley G, Rosen V, et al. The localized expression of extracellular matrix components in healing tendon insertion sites: an in situ hybridization study. J Orthop Res. 2002;20(3):454-463.

33. Carpenter JE, Thomopoulos S, Soslowsky LJ. Animal models of tendon and ligament injuries for tissue engineering applications. Clin Orthop Relat Res. 1999;(367 Suppl):S296-311.

34. Chang J, Thunder R, Most D, Longaker MT, Lineaweaver WC Studies in flexor tendon wound healing: neutralizing antibody to TGF-beta1 increases postoperative range of motion. Plast Reconstr Surg. 2000;105(1):148-155.

35. Molloy T, Wang Y, Murrell G. The roles of growth factors in tendon and ligament healing. Sports Med. 2003;33(5):381-394.

36. Roberts A, Sporn M. The molecular and cellular biology of wound repair. In: Clark R, editor. 2nd ed1995;265-275.

37. Merino R, Ganan Y, Macias D, Economides AN, Sampath KT, Hurle JM. Morphogenesis of digits in the avian limb is controlled by FGFs, TGFbetas, and noggin through BMP signaling. Dev Biol. 1998;200(1):35-45.

38. Cowin AJ, Holmes TM, Brosnan P, Ferguson MW. Expression of TGF-beta and its receptors in murine fetal and adult dermal wounds. Eur J Dermatol. 2001;11(5):424-431.

39. Soo C, Beanes SR, Hu FY, et al. Ontogenetic transition in fetal wound transforming growth factor-beta regulation correlates with collagen organization. Am J Pathol. 2003;163(6):24592476.

40. Edwards SL, Lynch TS, Saltzman MD, Terry MA, Nuber GW. Biologic and pharmacologic augmentation of rotator cuff repairs. J Am Acad Orthop Surg. 2011;19(10):583-589.

41. Lee $\mathrm{EH}, \mathrm{Hui} \mathrm{JH}$. The potential of stem cells in orthopaedic surgery. J Bone Joint Surg Br. 2006;88(7):841-851.

42. Cook JL, Purdam CR. Is tendon pathology a continuum? A pathology model to explain the clinical presentation of load-induced tendinopathy. Br J Sports Med. 2009;43(6):409-416.

43. Abate M, Silbernagel KG, Siljeholm C, et al. Pathogenesis of tendinopathies: inflammation or degeneration? Arthritis Res Ther. 2009;11(3):235. 
44. Andres BM, Murrell GA. Treatment of tendinopathy: what works, what does not, and what is on the horizon. Clin Orthop Relat Res. 2008;466(7):1539-1554.

45. Alfredson $\mathrm{H}$, Forsgren $\mathrm{S}$, Thorsen $\mathrm{K}$, Lorentzon R. In vivo microdialysis and immunohistochemical analyses of tendon tissue demonstrated high amounts of free glutamate and glutamate NMDAR1 receptors, but no signs of inflammation, in Jumper's knee. J Orthop Res. 2001;19(5):881-886.

46. Gulotta LV, Kovacevic D, Ehteshami JR, Dagher E, Packer JD, Rodeo SA. Application of bone marrow-derived mesenchymal stem cells in a rotator cuff repair model. Am J Sports Med. 2009;37(11):2126-2133.

47. Caplan AI, Dennis JE. Mesenchymal stem cells as trophic mediators. J Cell Biochem. 2006;98(5):1076-1084.

48. Pittenger MF, Mackay AM, Beck SC, et al. Multilineage potential of adult human mesenchymal stem cells. Science. 1999;284(5411):143-147.

49. Rodeo SA. Biologic augmentation of rotator cuff tendon repair. J Shoulder Elbow Surg. 2007;16(5 Suppl):S191-197.

50. Wurgler-Hauri CC, Dourte LM, Baradet TC, Williams GR, Soslowsky LJ. Temporal expression of 8 growth factors in tendon-to-bone healing in a rat supraspinatus model. J Shoulder Elbow Surg. 2007;16(5 Suppl):S198-203.
51. Oliva F, Via AG, Maffulli N. Role of growth factors in rotator cuff healing. Sports Med Arthrosc. 2011;19(3):218-226.

52. Uggen C, Dines J, McGarry M, Grande D, Lee T, Limpisvasti $\mathrm{O}$. The effect of recombinant human platelet-derived growth factor BB-coated sutures on rotator cuff healing in a sheep model. Arthroscopy. 2010;26(11):1456-1462.

53. Manning CN, Kim HM, Sakiyama-Elbert S, Galatz LM, Havlioglu N, Thomopoulos S. Sustained delivery of transforming growth factor beta three enhances tendon-to-bone healing in a rat model. J Orthop Res. 2011;29(7):1099-1105.

54. Kim HM, Galatz LM, Das R, Havlioglu N, Rothermich SY, Thomopoulos $\mathrm{S}$. The role of transforming growth factor beta isoforms in tendon-to-bone healing. Connect Tissue Res. 2011;52(2):87-98.

55. Kovacevic D, Fox AJ, Bedi A, et al. Calcium-phosphate matrix with or without TGF-beta3 improves tendon-bone healing after rotator cuff repair. Am J Sports Med. 2011;39(4):811-819.

56. Moffat KL, Kwei AS, Spalazzi JP, Doty SB, Levine WN, Lu HH. Novel nanofiber-based scaffold for rotator cuff repair and augmentation. Tissue Eng Part A. 2009;15(1):115-126.

57. Zhang X, Bogdanowicz D, Erisken C, Lee NM, Lu HH. Biomimetic scaffold design for functional and integrative tendon repair. J Shoulder Elbow Surg. 2012;21(2):266-277. 